\title{
Las organizaciones chinas en Colombia
}

\author{
Chinese organizations in Colombia
}

\author{
DIANA A. GÓMEZ* \\ LUZ M. DÍAZ**
}

RESUMEN: La existencia de organizaciones chinas en Colombia es relativamente reducida porque la inmigración china es reciente y no ha sido muy numerosa si se la compara con otros países de América Latina. Hasta la actualidad, no se han desarrollado investigaciones que aborden las características y evolución de las asociaciones de migrantes chinos establecidas en territorio colombiano. Tras una breve reseña de la inmigración china a Colombia, se abordan las características de esta inmigración china en la actualidad y los factores que han sido determinantes de ella entre los que se destacan la política inmigratoria colombiana; concomitante con ésta, la ausencia de una política exterior abierta y definida, y la situación de violencia interna que ha caracterizado a Colombia en las últimas siete décadas. En tal contexto, se realiza una caracterización y tipología de las organizaciones de inmigrantes chinos en Colombia.

PALABRAS CLAVE: migraciones internacionales, migraciones chinas, chinos de ultramar, organizaciones chinas en Colombia, China, América Latina.

ABSTRACT: The existence of Chinese organizations in Colombia is relatively small, since Chinese immigration is a recent phenomenon and has not been in great quantities, if one compares it to other Latin American countries. To date, no research initiatives have been carried out that tackle the characteristics and evolution of the Chinese migrant associations founded in Colombian territory. Beginning with a brief summary of Chinese immigration to Colombia, the study looks at the characteristics of this Chinese immigration as it is today, and the factors which have been determinant in its development, among them of particular interest is the Colombian migration policy; concurrently, the absence of a clearly defined foreign policy; and the context of internal violence which has characterized Colombia through the past seven decades. In this context, the article attempts to develop a characterization and typology of Chinese immigrant organizations in Colombia. KEY wORDS: international migration, Chinese migration, overseas Chinese, Chinese organizations in Colombia, China, Latin America.

\footnotetext{
* Profesora Asociada Instituto de Estudios Urbanos, Universidad Nacional de Colombia, con línea de investigación en Gobierno Urbano y Relaciones Internacionales. Directora de la Cátedra China, Universidad Nacional de Colombia.

** Experta en Migraciones Laborales Internacionales e Instituciones en América Latina.
} 


\section{INTRODUCCIÓN}

E ste artículo representa una reflexión inicial sobre el papel de las organizaciones de las comunidades de inmigrantes chinos en Colombia. Hasta la actualidad, no se han desarrollado investigaciones que aborden las características y evolución de las asociaciones de migrantes chinos establecidas en territorio colombiano y su relación con la vida social, política y económica de las ciudades que los han acogido. El presente estudio resulta de interés para comprender diversas características y aspectos nodales de la comunidad china en Colombia, sus momentos históricos más relevantes y los perfiles de sus organizaciones en el momento actual.

\section{Metodología}

Esta investigación hace parte de los esfuerzos realizados por el Centro de Migración y Desarrollo de la Universidad de Princeton y la Universidad de Miami bajo la coordinación de los profesores Alejandro Portes y Ariel Armony. Para realizar el presente estudio se desarrolló una estrategia metodológica que incluyó: 1) aplicación de 10 entrevistas semiestructuradas de un cuestionario para líderes de organizaciones; ${ }^{1}$ 2) observación y acción participativa en diferentes escenarios formales e informales; 3 ) conversaciones formales e informales con miembros de la comunidad china, empresarios y funcionarios que fueron relevantes para comprender las características de esta comunidad y sus formas de asociación; 4) elaboración de un directorio de asociaciones así como de entidades, empresas, centros culturales chinos y/o relacionados con China establecidos en Colombia.

${ }^{1}$ El formato utilizado por este proyecto fue adaptado a las condiciones específicas de las instituciones de inmigrantes chinos en Colombia.

$76 \frac{\text { PRIMER SEMESTRE } 2016}{\text { MIGRACIÓN Y DESARROLLO NÚM. } 26}$ 
Es importante resaltar que el estudio se logró gracias al conocimiento y los vínculos que Centro China ${ }^{2}$ tiene desde hace casi una década con la comunidad china, académicos, funcionarios del gobierno colombiano y empresas que vinculan a Colombia con China. Así mismo, diversas publicaciones realizadas anteriormente son antecedentes relevantes que permitieron la realización del proyecto.

\section{Reseña de la historia de la migración china a Colombia}

Los primeros inmigrantes chinos arribaron a territorio colombiano en 1854 para las labores de construcción del ferrocarril que atravesaría posteriormente el istmo, en la provincia de Panamá (que en aquella época pertenecía a Colombia), porque la mano de obra proveniente de otros países no era suficiente (Patiño, 2006). Procedían de la provincia de Cantón y ya habían trabajado en las labores del Pacific Railroad en la costa oeste de Estados Unidos. Sin duda, la descripción de Patiño (2006) es patética:

... Llegaron el 30 de marzo de 1854 y desfilaron por la ciudad capital hasta los barracones en los que serían alojados y en los que pasaron una miseria existencia. La traída de los chinos fue una medida desesperada de los administradores de la empresa ferroviaria, que no encontraba suficientes trabajadores entre la población nativa y que había fracasado con la «importación» de mano de obra irlandesa, alemana y de otros países europeos.

Los chinos fueron objeto de hostilidades por parte de los irlandeses que aún quedaban en Panamá y ocuparon trabajos muy rudos. Muchos de ellos se hundieron en una situación de depresión que afectó su salud y su rendimiento laboral. Su comportamiento llegó a extremos como lo que ocurrió en un lugar llamado Matachín, que era una de las muchas estaciones intermedias establecidas a lo largo del ferrocarril. Allí se produjo un suicidio masivo

${ }^{2}$ Centro China, el Centro de Estudios de China en Colombia, es una entidad académica privada. 
de trabajadores chinos que alarmó a las autoridades locales y les llevó a tomar medidas que aliviaran las condiciones de vida de estos emigrantes, según lo relata Patiño en su artículo.

Pero pese a las adversidades, los malos tratos, al clima malsano y los fallecimientos, un buen número de chinos logró culminar las tareas ferroviarias y establecerse en Panamá, a donde llevaron posteriormente sus familias y en donde prosperaron significativamente. Sin embargo, dadas las penosas condiciones en Panamá, una notable cantidad de estos inmigrantes, se trasladaron a otras ciudades de Colombia, particularmente Barranquilla, Buenaventura y Cartagena, buscando nuevas oportunidades. El premio Nobel Gabriel García Márquez, a través de sus relatos en la novela El amor en los tiempos del cólera hace una excelente semblanza sobre la vida de los chinos que llegaron a Barranquilla, en el Caribe colombiano, durante el siglo XIx:

Habían llegado a fines del siglo anterior huyendo del flagelo de la fiebre amarilla que asoló a Panamá durante la construcción del ferrocarril de los dos océanos, junto con muchos otros que aquí se quedaron hasta morir, viviendo en chino, proliferando en chino, y tan parecidos los unos a los otros que nadie podía distinguirlos. Al principio no eran más de diez, algunos de ellos con sus mujeres y sus niños y sus perros de comer, pero en pocos años desbordaron cuatro callejones de los arrabales del puerto con nuevos chinos intempestivos que entraban en el país sin dejar rastro en los registros de aduana.

Algunos de los jóvenes se convirtieron en patriarcas venerables con tanta premura, que nadie se explicaba cómo habían tenido tiempo de envejecer. La intuición popular los dividió en dos clases: los chinos malos y los chinos buenos. Los malos eran los de las fondas lúgubres del puerto, donde lo mismo se comía como un rey o se moría de repente en la mesa frente a un plato de rata con girasoles, y de las cuales se sospechaba que no eran sino mamparas de la trata de blancas y el tráfico de todo. Los buenos eran los chinos de las lavanderías, herederos de una ciencia sagrada, que devolvían las camisas más limpias que si fueran nuevas, con los cuellos y los puños como hostias recién planchadas. Fue uno de estos chinos buenos el que derrotó en los Juegos Florales a setenta y dos rivales bien pertrechados. Nadie entendió el nombre cuando Fermina Daza lo leyó ofuscada. 
No sólo porque era un nombre insólito, sino porque de todos modos nadie sabía a ciencia cierta cómo se llamaban los chinos. (García Márquez, citado por Checa-Artasu, 2007).

En efecto, a principios del siglo xx, Barranquilla, que ya era una activa ciudad y en pleno crecimiento, con oportunidades comerciales y de trabajo, se constituyó en el principal destino de la inmigración china. Las familias chinas radicadas allí se dedicaron a la siembra de verduras, al comercio al detalle y a áreas de servicios, especialmente en el campo de restaurantes y lavanderías. Prosperaron y se agruparon en el único «barrio chino» que ha existido en cualquiera de las ciudades colombianas. Desde Barranquilla los descendientes de los chinos incursionaron poco a poco en otras ciudades del Caribe colombiano durante el siglo xx.

Otro destino en el territorio colombiano de los chinos que vinieron desde Panamá fue la ciudad de Buenaventura, ubicada sobre el Pacífico colombiano y principal puerto de arribo para los viajeros de países del Oriente. En efecto, por aquella época, el general Tomás Cipriano de Mosquera logró que el Congreso de la República aprobara la importación de 1,000 chinos para que trabajaran en el entonces «camino de ruedas a Buenaventura», siguiendo el ejemplo de los constructores del Ferrocarril de Panamá (Patiño, 2006). Desde Buenaventura, los chinos se asentaron en el departamento del Valle del Cauca, ubicado al sudoccidente de Colombia, y es por ello que hoy se encuentran en esta región numerosos restaurantes chinos, generalmente de cocina cantonesa. Sólo más tarde llegaron a Bogotá, y esto sucedió de manera subrepticia a partir de la década de los veinte del siglo pasado. En los años cincuenta empiezan a registrarse los primeros restaurantes chinos en la capital, según lo reseña Lácydes Moreno (Patiño, 2006), así como pequeñas tiendas de comercio al detalle. Una parte corresponde a la migración interna de chinos hacia Bogotá y la otra se debe a la llegada de chinos procedentes en su mayoría de la provincia de Guandong, producto de la instauración del gobierno comunista en 1949, lo que generó la emigración de chinos simpatizantes del partido nacionalista Kuomintang (Gómez, 2007). 
Durante el siglo xx, vale anotar que, desde China continental, el gobierno diseñó diversos programas que fomentaban la emigración por un periodo determinado y con fines específicos. Es así como muchos de los chinos que hoy tienen restaurantes, vinieron en principio con el «Programa de Hoteles y Restaurantes» del gobierno chino. Este programa consistía en permanecer en un país por un periodo de dos o tres años para fomentar la industria culinaria. Ellos no escogían el país de destino, era el gobierno quien les asignaba un país y allí eran enviados (Marriaga, 2007).

Fue sólo hasta hace menos de diez años cuando los inmigrantes chinos empiezan a tener una presencia significativa por la llegada de empresas chinas que se han instalado en Colombia gracias al proceso de reforma y apertura chinos. Pero la inmigración de ciudadanos chinos de manera creciente a territorio colombiano no ha estado exenta de dificultades. Un líder y ejecutivo de una empresa transnacional china (ETN) nos refería en una de las entrevistas que:

[...] en este momento se ha incrementado la llegada de chinos y siguen llegando porque les interesan mucho acá los hidrocarburos. Se está construyendo la represa de Monte Líbano; la está haciendo una empresa que se llama Gecelca y allí vinieron muchos chinos. Los trabajadores colombianos nos han hecho muchas huelgas porque plantean que hay muchos chinos en el desarrollo del proyecto y que eso impide generar empleo para los colombianos y pagarles iguales salarios que a los chinos... De 2.500 trabajadores que se necesitaban para el proyecto, se trajeron 250 chinos pero existió mucha traba para que los dejaran venir. A nosotros nos tocó demostrar que aquí no había ingenieros de baños, que no había ingenieros de unas turbinas que necesitaban operar, para que los dejaran entrar.

A este respecto, vale anotar que si bien existía anteriormente una normativa que definía un tratamiento diferenciado en términos de proporcionalidad entre trabajadores nacionales y extranjeros — con el fin de proteger el derecho al trabajo de los nacionales-, con la Ley 1429 del 2010 (artículos 63 y 65), se elimina esta diferencia, permitiendo que los extranjeros trabajen

$80 \frac{\text { PRIMER SEMESTRE } 2016}{\text { MIGRACIÓN Y DESARROLLO NÚM. } 26}$ 
en Colombia una vez que obtienen la visa, sin que los empleadores estén sometidos a la restricción de porcentajes de participación entre trabajadores colombianos y extranjeros.

\section{LA INMIGRACIÓN CHINA EN LA ACTUALIDAD}

En el caso colombiano se pueden establecer dos tipos de migrantes chinos. Aquellos que llegaron primero con un relativamente bajo perfil en términos de niveles educativos y socioeconómicos, configurándose como pequeños comerciantes, particularmente dedicados a pequeñas tiendas de ropa y a los tradicionales restaurantes. Y en segunda medida, quienes posteriormente han venido llegando para trabajar en las empresas transnacionales, que conforman una población de mayor impacto social, cultural y económico, con mayor nivel educativo, manejo del inglés (el idioma más utilizado para la comunicación con los colombianos) y mayor entrenamiento profesional y con un relativo menor perfil etario, es decir, que es una población más joven (mayoritariamente entre 30 y 40 años de edad, según se puede observar a través de nuestros contactos y entrevistas). Esta población llega para vivir en Colombia por un periodo de al menos cuatro años, que es el término máximo legalmente permitido en el marco de la Alianza Pacífico para las visas de negocios según la Ley 834 de 2013. Sin embargo, vale señalar que al vencimiento de este término es posible solicitar una prolongación de la visa.

Es cada vez más creciente la contratación de chinos por empresas de ese país recientemente instaladas en territorio nacional. Se trata de industrias particularmente del área petrolera, como Sinopec ${ }^{3}$ KeruiGroup, especializada en perforación de pozos petroleros y tuberías, y Sinochen, empresa petroquímica; ${ }^{4}$ de telecomunicaciones, como Huawei y ZTE; de la industria automotriz como Cinascar, y de la industria maderera, como Amboo, especializada en el manejo del bambú. Tal como lo señala Marriaga (2007), un

${ }^{3}$ Sinopec adquirió hace unos años por 400 millones de dólares a la compañía de hidrocarburos Onimex de Colombia y hoy es Mansarovar Energy Colombia.

${ }^{4}$ Sinochen invirtió 300 millones de dólares al comprar activos de la petrolera Emerald Energy. 
buen número de programas de infraestructura — específicamente vías, aeropuertos, puertos, y la infraestructura relacionada con la exploración y explotación de petróleo- emprendida en los últimos años, están asociados a la llegada de empresas chinas. Es el caso del conglomerado Capital Airport Holding, que maneja a través de Airplan los aeropuertos de las ciudades de Medellín, Rionegro, Carepa, Montería, Corozal y Quibdó. Después de tres años de iniciado el proyecto, la compañía estatal china Hydrochina Corporation en abril de 2014 hizo entrega oficial al Estado colombiano del «Plan Maestro de Aprovechamiento» del río Magdalena, el río más importante del país. Se trata de un amplio estudio consistente en un documento que alcanza 730 páginas sobre el manejo ambiental y las posibilidades de aprovechamiento comercial e industrial del Magdalena.

Es importante señalar que no existe una clara «distancia social» entre la comunidad china tradicional y los nuevos inmigrantes. La relación entre estos dos grupos es muy cercana, ya que, según lo observado, no establecen distancias. La primacía del bien común, la confianza, la concepción de «asociatividad» y lógica de lo colectivo está por encima de cualquier otra consideración. Ahora bien, no es fácil tener información muy exacta del número de inmigrantes chinos en Colombia. El último censo de población se realizó en 2005, apenas cuando se iba a iniciar el creciente flujo de inmigrantes vinculados a las empresas transnacionales. De hecho, el Censo Nacional de Población realizado en 2005 por el Departamento Administrativo Nacional de Estadísticas (DANE), señalaba que en más de 42 millones de habitantes del país, tan sólo 101,971 eran extranjeros, es decir, apenas 0.24 por ciento.

Se sabe que en 2008, según cifras de la Embajada China, residían en Colombia alrededor de 10,000 chinos (Gómez, 2008). Según el presidente de la colonia china, Kenny Tsui, el número de chinos residentes en Colombia en 2014 ascendió a cerca de 25,000. En la actualidad no existen cifras oficiales que den cuenta de la realidad de la inmigración china en Colombia. La información es muy parcial pero hace énfasis en el aumento de las cifras en los últimos años. Por ejemplo, Huawei, la empresa china de telecomunicaciones, tenía en 2007 unos 70 empleados chinos instalados por diferentes lapsos, en Bogotá; hoy día son cerca de 200. De igual manera, la empresa 
china productora de equipos de telecomunicaciones y soluciones en red, ZTE, llegó a Colombia en el 2004; ya en 2007 contaba con aproximadamente 30 nacionales chinos en su nómina (Marriaga, 2007) y en la actualidad tiene más de un centenar, según un ejecutivo de esta compañía.

Asimismo, los profesores de mandarín de los institutos «Confucio» y de colegios y universidades que imparten la enseñanza del idioma chino vienen a Colombia con programas del gobierno y de intercambio cultural similares a los que trajeron a los chefs a mediados del siglo xx, es decir, por un periodo acordado previamente. Pero «una vez están acá, hay unos que deciden quedarse por mucho más tiempo del previsto, como es el caso de Zhang Huijing, quien, como relata una colega, vino por un año y hoy, siete años después, continúa en Bogotá ejerciendo el cargo de vicedirectora del Instituto "Confucio», (Marriaga, 2007). Por lo general, estos chinos suelen no sólo establecerse en Colombia por tiempo extendido, sino también traer a amigos y familiares.

\section{FACTORES DETERMINANTES DE LA INMIGRACIÓN CHINA A COLOMBIA}

Conviene tener en cuenta algunos elementos claves que contribuyen a explicar la relativa baja presencia de extranjeros en el país a través de su historia y hasta el presente, particularmente de población de origen chino en comparación con otros países de la región. Es importante tener en cuenta que Colombia, a diferencia de otros países de América Latina como Venezuela, Argentina, Brasil y Perú, se ha caracterizado por albergar muy pocas comunidades de inmigrantes a lo largo de su historia, lo cual se vincula con la precaria apertura al exterior, razón por la cual históricamente ha recibido la denominación de "el Tíbet Latinoamericano». De esta tradición no escapan los flujos de población procedente de China. Esta situación se debe en gran medida a tres factores: 1) la política inmigratoria; 2) concomitante con ésta, la ausencia de una política exterior abierta y definida, y 3 ) el problema de la violencia que ha caracterizado a Colombia en las últimas siete décadas. 
Las siguientes líneas explican estos tres factores en relación con la inmigración china.

\section{1) La política inmigratoria colombiana}

La política inmigratoria colombiana fue excluyente y racista hasta bien entrado el siglo xx. En efecto, a lo largo del siglo pasado, claramente se prefería la inmigración europea, pues se creía que ésta traería prosperidad, desarrollo y ante todo "pureza de sangre». En 1914, el periódico de ideología conservadora, El Nuevo Tiempo, publicó lo siguiente: «Lo que nos debe preocupar no es traer inmigrantes sino impedir que lleguen negros, indios, chinos, japoneses y apaches» (Vallejo, 2006: 160, citado por Marriaga 2007). Incluso, en 1922 se expidió una ley que prohibía la entrada de indios y chinos al país.

Sólo hasta el 7 de febrero de 1980 se dio inicio a las relaciones oficiales entre China y Colombia, lo que ha influido también en el número relativamente reducido de población china en el país, según lo señala el académico y experto en las relaciones entre China y América Latina, Xu Shicheng (2014). En efecto, Colombia fue el país número catorce dentro de la región en formalizar relaciones diplomáticas bilaterales con China. Si bien durante buena parte del siglo xx la política inmigratoria colombiana se caracterizó, y aun hoy, por las trabas a todo aquel ciudadano de países como China que pretendiera ingresar al país, a inicios del presente siglo se presentó un giro inesperado: durante los años 2006 y 2007 se registró un salto mayúsculo en el número de inmigrantes chinos. Numerosos inmigrantes procedentes de la República Popular que viajaban con destino a Estados Unidos paulatinamente empezaron a utilizar a Colombia como trampolín para llegar a Norteamérica debido a que, en diciembre del 2006, se levantó la exigencia de visa para los chinos. Bajo el propósito de impulsar y desarrollar el turismo hacia Colombia, el gobierno colombiano decidió aminorar y simplificar los trámites y sólo bastaba con presentar pasaporte, tiquete de regreso y una justificación del viaje.

$84 \frac{\text { PRIMER SEMESTRE } 2016}{\text { MIGRACIÓN Y DESARROLLO NÚM. } 26}$ 
Cinco meses más tarde, el gobierno tuvo que revertir la medida, por cuanto el país empezó a llenarse de chinos ilegales. Pero el daño ya estaba hecho: durante el primer trimestre del 2007, arribaron 3,490 personas provenientes de China. En contraste, en el año anterior el número fue de sólo 850, porque estaba vigente la visa. Estos inmigrantes transitorios y fugaces eran con frecuencia alojados subrepticiamente en casas y lugares escondidos desde donde salían para continuar su viaje a Estados Unidos. El Departamento Administrativo de Seguridad (DAS), entidad encargada en esa época de vigilar el cumplimiento de las políticas de inmigración, encontró al menos dos rutas para el tráfico de personas: una que comenzaba en Beijing, cubriendo París y llegando hasta Bogotá. En esta ciudad los intermediarios conducían a sus cargas humanas hasta el puerto de Buenaventura y continuaban vía Panamá, Centroamérica y México hacia Estados Unidos. El otro itinerario partía de la capital colombiana hacia Cúcuta, en el oriente del país, para después adentrarse en territorio venezolano; de allí, se dirigían a Centroamérica y pasaban a México para llegar a territorio estadounidense (El Espectador, 2008). Al respecto, otro artículo de la Revista Semana (2007), describía así la situación:

Estos chinos no saben ni dónde están parados. A esa conclusión llegó el intérprete Willy Schafer cuando uno de los 101 jóvenes chinos hallados la semana pasada en Zipaquirá, le preguntó en mandarín cuál era su nacionalidad. Entonces Schafer le dijo que era de Colombia. «¿̇Y ese país dónde queda?» contra preguntó desconcertado el chino.

Los niños que vivían en la zona rural llamada Pasoancho, en la ciudad de Zipaquirá (próxima a la capital), fueron los primeros en advertir la presencia de los asiáticos:

Notaron que caras con ojos rasgados se asomaban inquietas por una ventana para verlos jugar. Con el paso de los días, los chinos fueron tomando confianza. Una tarde, luego de varias jornadas de sonrisas a través de la ventana, abrieron la puerta y pisaron la calle. Fue entonces cuando los moradores de Pasoancho se enteraron de que tenían por vecinos a 59 chinos hacinados en una casa vecina 
y a otros cuantos en otra casa también cercana. Pero no hubo alarma. Se trataba de personas amistosas que sólo querían distraerse jugando con los niños. Todos eran pésimos negociantes: cambiaban gustosos dólares y monedas chinas por un par de cigarrillos. Varias semanas de intercambio cultural pasaron antes de que el tema llegara a oídos de la Policía y el Departamento Nacional de Seguridad (Revista Semana, 2012).

Del total de estos chinos, apenas 21 tenían los papeles en regla. El intérprete Schafer, quien ayudó al DAS a entenderse con los forasteros, señalaba que son jóvenes de origen humilde, con educación básica y que manifestaban que venían de turismo al país, aunque apenas se movían alrededor de las casas donde fueron encontrados. Luego de este hallazgo, nuevos grupos fueron apareciendo en Bogotá, en viviendas precarias e improvisados galpones como alojamiento. Éstos eran generalmente jóvenes entre 20 y 30 años de edad, víctimas de redes de tráfico que les ofrecen llevarlos a Estados Unidos y Canadá. La mayoría de estos inmigrantes transitorios provenían de las provincias de Guangdong y Fujian:

Cuando llegan, los traficantes les quitan sus papeles y vienen convencidos de que la operación sólo tendrá éxito si se mantienen en silencio. Las investigaciones indican que las redes de tráfico de inmigrantes entran legalmente a los chinos por el aeropuerto El Dorado. Allí, un enlace se encarga de concentrarlos en casas alquiladas en donde deben esperar a que otra persona de la red los recoja para continuar su camino hacia Norteamérica (Revista Semana, 2007).

Por la misma época y con la no exigencia del requisito de visa para el ingreso a Colombia, el aeropuerto de París colapsó en cierto momento por tanta cantidad de chinos que iban llegando como lugar de escala para venir a Colombia, según nos lo relató en una entrevista Daniela Sánchez, directora ejecutiva de la Cámara Colombo-China de Comercio e Inversión. Según esta informante: «Frente a esta situación, el departamento colombiano de inmigración, encontró que había una montaña de inmigrantes que luego salían para Estados Unidos. Y aunque Estados Unidos tomó medidas con el

\footnotetext{
$86 \frac{\text { PRIMER SEMESTRE } 2016}{\text { MIGRACIÓN Y DESARROLLO NÚM. } 26}$
} 
gobierno colombiano para que eso no continuara, algunos se quedaron [...]» Interesa anotar que algunos sectores de población joven continuaron llegando y quedándose en Colombia utilizando las relaciones de parentesco y/o redes sociales de apoyo.

2) La existencia de una política exterior fragmentada y contradictoria

La política exterior colombiana es fragmentada, contradictoria y esto se refleja fácilmente en la falta de claridad en la normatividad para la población inmigrante o que aspira a inmigrar a Colombia, lo cual impacta en el tema de la expedición de visas para extranjeros. Mientras existe una política de apertura a la inversión extranjera y una legislación que les otorga garantías, en la cancillería (Ministerio de Relaciones Exteriores) se han instaurado una serie de trámites engorrosos para el ingreso de empresarios, tecnólogos, trabajadores temporales, y también de académicos, expertos y visitantes invitados a congresos y encuentros de alto nivel, según se puede deducir del Decreto 834 de 2013 sobre «Ingreso, Permanencia y Salida de los Extranjeros del Territorio Nacional Colombiano».

De otra parte, «[...] aún se sigue prefiriendo países como Estados Unidos o Europa para que vengan a invertir y de alguna forma la estructura política es muy similar con estos países, con China no tanto, por eso hay mayor dificultad para que la inversión sea aún mayor», dice Daniela Sánchez. Así mismo, agregaba que:

En mi trabajo diario, una de las principales quejas para que la inversión china sea mayor, el obstáculo principal, es migratorio, no importa qué nivel o tipo de inversión estén buscando los chinos. El gobierno de Colombia es bastante estricto para dejarlos entrar incluso cuando ya están acá, para dejarlos extender su visa. Es tan grave que ya la embajadora de Colombia en China está cuestionando esa situación que se viene acentuando en los últimos años (Entrevista, 2014). 
Los empresarios chinos también señalan que:

Colombia necesita más inversión, nosotros como empresarios nos interesan los negocios, para muchos empresarios chinos el cumplimiento es vital, aún tenemos muchos problemas de visa, entonces esto daña la relación con el país. A futuro queremos ser socios estratégicos del gobierno, pero no sé cuál es la idea de Colombia, como cuando uno pide matrimonio y no le contestan (risas) [...] Pero hay muchas empresas chinas y colombianas que estamos trabajando duro para triunfar en este proceso (Entrevista, 2014).

Nótese que lo anterior entra en abierta contradicción con las políticas de desarrollo de los gobiernos de Uribe (2002-2010) y Santos (2010-2014), reelegido éste último en 2014, que abrieron un espacio favorable a la inversión internacional de suerte que las empresas transnacionales en el campo minero-energético, automotriz y de telecomunicaciones, se ven estimuladas para la inversión en Colombia. Cabe recordar que una de las políticas del gobierno de Santos durante su primer periodo de gobierno fue el impulso a lo que ha llamado «la locomotora minero-energética» que es una estrategia de desarrollo, caracterizada por impulsar la explotación de hidrocarburos, dando amplias facilidades a las empresas multinacionales que estén en condiciones de invertir en tales proyectos. En términos generales, la relación de Colombia con China ha aumentado de una manera muy cautelosa. En el año 2005, con la visita del presidente Álvaro Uribe a China y en 2012 con la de Juan Manuel Santos al país asiático, se logró la firma de acuerdos y actividades multitemáticas, no obstante, magros han sido los resultados.

\section{3) El fenómeno de la violencia}

Colombia es un país en donde los conflictos armados han perdurado por más de 70 años y esta situación ha permeado y ha incidido en todos los hechos sociales, la cultura, la economía y la política, así como en las condiciones de la inmigración internacional, ya que para muchos sectores potencialmente

\footnotetext{
$88 \frac{\text { PRIMER SEMESTRE } 2016}{\text { MIGRACIÓN Y DESARROLLO NÚM. } 26}$
} 
migrantes no se tendrían las suficientes condiciones de seguridad para instalarse en el país. Por otra parte, el conflicto interno colombiano, unido a la muy heterogénea geografía y la histórica fragmentación por regiones claramente diferenciadas (costa Caribe, zona Andina, costa Pacífica, Orinoquía y Amazonía) ha impedido el desarrollo de la infraestructura a lo largo y ancho del territorio colombiano. Esto ha impactado grandemente a zonas como la costa Pacífica colombiana, hoy día completamente abandonada por el Estado.

Los efectos del conflicto armado en Colombia como obstaculizadores de actividades de los inmigrantes aparecen de manera reiterativa en las entrevistas realizadas para este estudio. Un líder de la comunidad china nos manifestaba: «el único problema de Colombia es la paz, de resto, perfecto, es un paraíso [...]». Otro ejecutivo de una empresa multinacional instalada en Colombia se refería al problema en los siguientes términos:

[...] Ahorita el mayor problema es con las FARC ${ }^{5}[\ldots]$ como nunca colaboramos con ellos, porque ellos nos amenazan, y nunca pagamos nada y como tenemos mucha protección, siempre nos molestan. Afortunadamente tenemos este convenio con el Ministerio de Defensa de Colombia, tenemos 300 soldados apoyándonos, sólo en el departamento del Caquetá.

Otro líder chino señaló:

Queremos llegar e invertir en Colombia, pero żcuáles son los incentivos para venir si hay problemas de guerrilla? Hace cuatro años secuestraron cuatro chinos de la compañía multinacional petrolera Emerald Energy, dos años después fueron liberados, entonces allí se tranca todo, porque si yo tengo que invertir como empresario, hay problema de seguridad. En Perú no hay problema de guerrilla y en Argentina tampoco hay. Por eso es muy importante si este gobierno logra la paz con guerrilla y deje sin conflicto armado al país, entonces Colombia será prioritaria. Pero hasta ahora es complicado por la inseguridad.

${ }^{5}$ Las farc son las Fuerzas Armadas Revolucionarias de Colombia, una guerrilla que ha estado combatiendo por más de 50 años. 


\section{Caracterización y tipología de las organizaciones}

En este estudio se consideró como «organización» a toda agrupación que estuviera organizada formal o informalmente con el objetivo de realizar actividades de carácter cultural, educativo, político o económico, entre otros, y que incluyera entre sus objetivos formales o informales funciones para con los inmigrantes chinos. Las organizaciones existentes en Colombia son la Organización de la Colonia China en Colombia, la Asociación de Empresas con Inversiones Chinas en Colombia, la Alianza Colombo-China, Centro China, los tres institutos "Confucio» y las tres aulas «Confucio», la Asociación de Amistad Colombo-China, la Organización para el Fomento de las Relaciones Colombia-China, la Cámara de Comercio e Integración Colombo-China, la Cámara de Comercio e Inversión Colombo-China y la Organización de los Chinos de Ultramar en América Latina, esta última desborda las fronteras nacionales pero da cuenta de la dinámica de las asociaciones chinas en Colombia.

Las organizaciones se discriminaron según su naturaleza y funciones en cinco tipos, a saber: 1) asociaciones de la comunidad inmigrante china: la Organización de la Colonia China en Colombia, la Asociación de Empresas con Inversiones Chinas en Colombia y la Organización de los Chinos de Ultramar en América Latina; 2) entidades creadas a iniciativa del gobierno chino: aulas "Confucio» e institutos "Confucio»; 3) entidades culturales y académicas creadas por chinos y colombianos: Centro China y la Alianza Colombo-China; 4) Asociaciones de Amistad Colombo-China creadas por colombianos: Asociación de la Amistad Colombo-China y Organización para el Fomento de las Relaciones Colombo-Chinas, y 5) Cámaras de Comercio Binacionales creadas a iniciativa de empresarios colombianos y chinos: Cámara de Comercio e Integración Colombo-China y Cámara de Comercio e Inversión Colombo-China.

La organización más antigua data de 1977, cuando fue creada la Asociación de la Amistad Colombo-China, le sigue la Cámara de Comercio e Integración Colombo-China, mientras que las demás existen desde hace menos de diez años. Las asociaciones más importantes y tradicionales se ubican en

$90 \frac{\text { PRIMER SEMESTRE } 2016}{\text { MIGRACIÓN Y DESARROLLO NÚM. } 26}$ 
el norte de la ciudad, en uno de los sectores más selectos del área El Chicó, las demás se encuentran en sectores aledaños o cercanos a éste. En general, se trata de organizaciones que combinan, en mayor o menor medida, actividades económicas, culturales y sociales. Una de las cámaras de comercio desarrolla actividades culturales, así como la mayoría también realiza actividades académicas y educativas, y en su totalidad desarrollan actividades sociales. No obstante, cabe llamar la atención sobre el hecho de que las organizaciones conformadas por población netamente china se caracterizan por dos factores: el desarrollo de actividades solidarias que benefician no sólo a la comunidad china, sino también a Colombia y, de otra parte, porque sus actividades sociales se realizan de manera muy endogámica.

Los procesos de toma de decisiones son muy variables entre las organizaciones, pero en su gran mayoría las juntas directivas o comités tienen un peso relevante. En una, en cambio, las decisiones las toma el líder, a veces asesorado por personas externas a la organización, otras veces por personas de gran trayectoria y prestigio, como los «ancianos». Una de las cámaras, la de Inversión, es la que mantiene más vínculos con representantes de ambos poderes en ambos países. Dado que la comunidad china en Colombia no es muy grande, casi todas las organizaciones se conocen entre sí, pero vale destacar que la más grande, más multifuncional y de mayor contacto con las demás es la de la Colonia China. Respecto a las demás, las asociaciones de Amistad y la Cámara de Comercio e Inversión son bastante cercanas a la Embajada China en Colombia.

El transnacionalismo quizá es más marcado en el caso de la Cámara de Comercio e Integración, la cual tiene permanente contacto transnacional con China y sus ocho oficinas en diferentes ciudades del territorio chino. Conviene citar una frase del presidente de la Colonia China en Colombia, cuando señala:

Asociarse, es parte de la cultura china. Los chinos se reúnen tanto como lo hacen los judíos por fuera de Israel y en todas partes del mundo, pero diferente a ellos, no hablan tanto de negocios, pero sí hablan de crecimiento de la comunidad china, de ayuda de uno con el otro, o sea ellos pueden estar viviendo aquí, inclusive 
ya nacionalizados como colombianos, pero igual viven con un amor de país, a la patria de sus padres, la mayoría todavía vive con ese pensamiento.

Mientras la comunidad china se reúne para celebrar de manera privada la Fiesta de Primavera o Nuevo Año Chino, Centro China y la Alianza Colombo-China son las únicas entidades que organizan hoy día la celebración para público colombiano. A continuación haremos una breve reseña de cada una de las organizaciones:

\section{ASOCIACIONES DE LA COMUNIDAD INMIGRANTE CHINA}

\section{1) La Organización de la Colonia China en Colombia}

Esta organización empieza a consolidarse en el presente siglo. Antes no existía una entidad como tal, de acuerdo con el testimonio de su presidente, Kenny Tsui, quien relata en una entrevista lo siguiente:

Yo me posesioné como presidente en el 2001 y no había una organización como tal a nivel nacional. Trabajé para lograrlo, entonces ya la gente me dio mucha confianza. Empezaron a llamarme de todas aquellas ciudades y municipios que no tenían organización entre ellos, porque querían afiliarse con nosotros. La colonia se volvió una cuestión nacional.

La Colonia China reúne a los representantes de las ciudades asociadas de Colombia, en Bogotá, cada dos meses y cada seis meses convocan una conferencia entre todos los líderes de diferentes ciudades. Las organizaciones locales que se agrupan dentro de la Colonia China son entre otras: Barranquilla, Cali, Medellín, Neiva, Cartagena, Montería, Bucaramanga y Bogotá.

Rápidamente el presidente de la Colonia logró abarcar el ámbito nacional: «Yo estoy como Presidente de toda la Organización, en Medellín tengo mi gente, tengo mi representante en Cali, tengo representantes en todos

$92 \frac{\text { PRIMER SEMESTRE } 2016}{\text { MIGRACIÓN Y DESARROLLO NÚM. } 26}$ 
lados, entonces ellos me reportan». Kenny explica que el trabajo de la organización es preponderantemente de tipo solidario:

Hay un trabajo muy importante que consiste en la ayuda que prodigamos a nuestros nacionales. Hay mucho chino recién llegado que no habla el idioma y que tiene complicaciones con su visa. Todo el trabajo es voluntario, lo sacamos a través de las colectas y nos ayudamos. O sea que si encontramos un chino tan enfermo que no tiene para ir a un médico, pues le hacemos donación. Eso es lo lindo que hacemos nosotros, no dejamos un ciudadano chino sufriendo de hambre, que no tenga para el médico, para el estudio, para el entierro, entonces el objetivo nuestro es pensar en el bienestar de todos los chinos que vienen a Colombia, que se sientan ayudados. Este es un trabajo que venimos haciendo hace 14 años y hemos logrado muy buenos resultados y mucha confianza.

Otra actividad desarrollada han sido los cursos de idioma chino para los hijos de los inmigrantes, actividad que llegó a ser financiada por el presidente de la Organización, quien es también un empresario muy reconocido dentro de la Colonia China. Al referirse a sus actividades con la población colombiana dice: «Además de lo que hacemos con nuestros chinos, pensamos en las actividades de beneficencia en Colombia. Desde que yo me posesioné y a partir del cuarto año, hemos estado repartiendo juguetes todos los fines de año. Llevamos regalos navideños a diferentes municipios, en Bogotá, Chía, Soacha, Tibacuy, a los niños pobres colombianos». Y continúa diciendo:

[...] hace cinco años cuando recién se posesionó Santos por primera vez como Presidente, hubo serios problemas de inundaciones en Colombia ìrecuerda? Nosotros alcanzamos a hacer una colecta de $\$ 80^{\prime} 000,000$ de pesos (alrededor de 40 mil dólares en ese momento). Eso no lo hace ninguna colonia ya sea española, judía o japonesa. Ninguno ha hecho alguna cosa así para beneficiar al pueblo colombiano. Pero lo hacemos nosotros, porque yo siempre vengo diciendo a todos los chinos que viven acá: "Usted tiene que contribuir a la comunidad donde vive, unirse a ellos, generar la solidaridad entre la gente, la comunidad de usted es muy importante, pero también hay que pensar en la comunidad colombiana, donde están ustedes [...] porque un restaurante żdónde está haciendo 
la plata? ¿Quién está comprando? ¿Cuáles son los clientes suyos? [...] colombianos».

Agrega que:

[...] la comunidad china en Colombia está muy unida, incluso nos han considerado como uno de los mejores ejemplos de América Latina. En el caso del terremoto 2008, usted no se imagina, yo empecé a hacer recolecta, esto no lo hace nadie para apoyar a los damnificados del terremoto de Sichuan, donde murieron 105,000 chinos. Hicimos una colecta de 115,000 dólares que es muy alta si se tiene en cuenta que sólo viven aquí 25,000 chinos. Li Chang Hua (el embajador chino en Colombia en esa época) estaba feliz y no hace sino decirle a todo el mundo lo que hemos logrado, porque se sentía orgulloso como embajador aquí.

Una actividad asidua de la Organización de la Colonia China con sus compatriotas es el acompañamiento en asuntos legales referidos a las autoridades colombianas, puesto que la mayoría de los chinos no hablan español, entonces la Organización dispone de un servicio de traductor al español. En los juzgados, por ejemplo, el traductor ayuda a explicar a los jueces y a los fiscales sobre la cultura china y a los chinos sobre la justicia y la ley colombiana cuando ellos la desconocen. En las actividades cotidianas es muy corriente mantener elementos claves de su cultura:

Nosotros conservamos la comida y las tradiciones. Los niños también lo hacen, las hijas mías comen con palitos en la casa; claro que salimos a McDonald's, a todas partes. Pero igual, mantenemos esa cultura de comer en la casa, de reunirnos nosotros, en familia. Espero que esto se pueda mantener. Igual ellos son de origen chino, mi papá es chino, el papá de mi señora también es chino, no quiero que llegue un día en que yo pierda esa costumbre. Mis hijas pueden ser ciudadanas y sentirse orgullosas de ser ciudadanas americanas (sus hijas nacieron y residen en Puerto Rico y son ciudadanas de Estados Unidos) y tener sus logros profesionales en el futuro, pero hay que saber algo de su origen, de sus padres, de sus abuelos, eso es muy importante.

$94 \frac{\text { PRIMER SEMESTRE } 2016}{\text { MIGRACIÓN Y DESARROLLO NÚM. } 26}$ 
En tal sentido, cabe destacar las labores que realiza el propio gobierno chino en pro de sus connacionales emigrados. Como lo relata Kenny,

El gobierno chino ha ayudado mucho. El Consejo de Estado del gobierno chino invita cada dos años a una conferencia. Este año yo envié un par de representantes míos porque yo estaba súper ocupado para asistir. A nivel mundial todos los líderes de la comunidad china participan en una conferencia bianual en China y el presidente Xi Jinping los recibe y habla de logros económicos de China, habla de todo. El presidente chino le da el mensaje de que usted aunque viva en otro país diferente a China, usted cuenta con el respaldo de la patria. Aunque sus hijos hayan nacido en el extranjero y vivan con la cultura de allá, en el futuro puede contar con esta cultura. El gobierno chino cada año organiza campamentos para los niños de segunda y tercera generación, este año mis dos hijas están participando, es un campamento en la provincia de Jiangsu donde ven aspectos culturales y folclóricos de China.

Estos eventos son financiados por el gobierno chino y buscan afianzar las relaciones culturales de sus niños en el exterior con su cultura y sus tradiciones.

En la Organización de la Colonia China en Colombia, se evidencian los nexos de parentesco, el arraigado sentido de familia transmitido mediante la tradición confuciana, así como los lazos de origen territorial, su habilidad de trabajar en equipo, y la construcción de confianza como uno de sus principios rectores y orientadores de sus comportamientos cotidianos.

\section{2) Asociación de Empresas con Inversiones Chinas en Colombia}

Esta asociación fue creada el 18 de junio de 2010 por 16 empresas: Sinopec Internacional Petroleum Exploration \& Production Corp., Huawei Technology, ZTE Corporation, Hidrochina Internacional, Emerald Energy, Kerui Group, Banco de Desarrollo de China, China International Water \& Electric Corp., Sinohidro Corporation, Sinopec International Petroleum Service, 
China Harbour, Grupo Langsha, Amboo Ltda., Pan-Pacific Andina Ltda., East Industry Ltda. y Latin Comercio. Ellas ratificaron el establecimiento de esta organización no lucrativa formada voluntariamente por sucursales de grandes empresas transnacionales chinas con inversiones en Colombia, en representación y salvaguardia de sus intereses y derechos y para coordinar sus relaciones. La Junta Directiva interina es liderada por el señor Ye Hui Min, presidente de Sinopec Internacional Petroleum Exploration \& Production Corp., Sucursal Colombia.

Un alto funcionario de la empresa Emerald, nos señala que:

Tenemos una organización de empresarios chinos registrada en la embajada china, pero es para compartir informaciones, para mejorar la amistad, nada más, no tenemos muchos servicios. Esta organización de empresarios chinos en Colombia, existe más o menos desde el 2010, pero agrupa sólo los empresarios grandes: Emerald Energy, Huawei, ZTE, Sinopec, entre otras. Actualmente, la presencia más poderosa de empresarios chinos en Colombia está en los hidrocarburos y las comunicaciones, y el resto son pequeños empresarios de la construcción, automóviles y una empresa colombiana socia de empresas chinas.

Las personas que vienen desde China contratadas para trabajar con las empresas transnacionales, tienen generalmente alojamiento y alimentación organizada por la empresa contratante (almuerzan dentro de la empresa y suelen vivir muy cerca de ella); permanecen por un periodo de 3 a 4 años renovables y están muy ligadas a su trabajo y a sus compañeros. Esto se hace viable por principios característicos de su cultura como la importancia de trabajar en equipo, los cuales se refuerzan entre los chinos de ultramar. Los chinos de ultramar se identifican a partir de su mismo origen regional, local, o provincial, la identificación con un dialecto común y los lazos de asociación surgidos de prácticas y habilidades compartidas. Asimismo, la confianza derivada de la recomendación y las experiencias previas es un factor fundamental para las operaciones de las empresas.

Sin embargo, dadas las enormes diferencias culturales con el país receptor, las entrevistas revelan el grado de endogamia de la comunidad china. A

$96 \frac{\text { PRIMER SEMESTRE } 2016}{\text { MIGRACIÓN Y DESARROLLO NÚM. } 26}$ 
continuación transcribimos las palabras de un ejecutivo chino que lleva 8 años viviendo en Colombia que reflejan en buena medida actitudes que también fueron reiterativas en otras entrevistas:

De acuerdo con mi experiencia en Colombia, debo decir que a nosotros nos falta mucho conocimiento de cultura latina, entonces siempre necesitamos una guía para apoyarnos, para ayudarnos, para conocer este continente, este mercado es muy, muy importante, la idea es que los chinos deben aprender de los colombianos cómo piensan los colombianos y los latinos, cómo funcionan las reglas, los juegos de negocio acá, cómo podemos ganar más mercado, cuál es el requisito, la meta de los consumidores. Hay mucha cosa para aprender y sobre todo también la actitud para la vida, acá es mejor, los chinos deben aprender más cómo disfrutar la vida, no sólo trabajar, eso es importante como humanos. Trabajar, żpara qué? Aquí nos damos cuenta que también trabajamos para la familia, para la vida. Trabajar no es para los jefes, y así aprendemos de los colombianos.

\section{3) La Organización de los Chinos de Ultramar en América Latina}

Los chinos en Colombia participan en una organización coordinada entre connacionales que residen en todos los países de Centro y Sudamérica y realizan encuentros anuales.

El año pasado se reunieron en Ecuador, el antepasado en Panamá y este año en Perú $[\ldots]$ y todo el mundo nos dice que quiere esa importante reunión en Colombia. Pero su presidente, que es chino residente en Colombia, dice que una vez el presidente Santos firme la paz con la guerrilla los trae. Son como quinientas personas y no sólo participan los ciudadanos chinos de ultramar, también vienen representantes del gobierno chino. Como esta es una organización de chinos de ultramar, el gobierno está muy pendiente de lo que hacemos nosotros, para saber, para apoyar, para que no haya una organización antigobierno; por eso ellos participan [...] (Entrevista a dirigente chino, 2014). 


\section{ENTIDADES CREADAS A INICIATIVA DEL GOBIERNO CHINO}

\section{1) El Instituto Confucio}

Como es bien sabido, el objetivo del Instituto "Confucio», como en otros lugares del mundo, es traer la milenaria cultura china a Colombia, dar a conocer su tradición histórica, promover el conocimiento del idioma y la cultura de China en Colombia y ofrecer una plataforma más amplia para el fomento del intercambio cultural entre los dos países. En Colombia existen tres institutos «Confucio» que desarrollan actividades a lo largo del año. Fueron establecidos en la Universidad de Los Andes en 2007 (Bogotá), en Eafit en 2010 (Medellín) y en la Universidad «Jorge Tadeo Lozano» en 2013 (Bogotá), los cuales siguen las mismas políticas de similares institutos en todo el mundo. Su personal es mitad chino y mitad colombiano. Entre ellos no se manifiestan conflictos destacados hasta el momento actual.

\section{2) El Aula Confucio}

En Colombia también existe el Aula "Confucio», la primera fue creada en el Colegio Nueva Granada de Bogotá, mediante un convenio con un colegio en China, en mayo de 2009. Su director educativo es Kevin Zhang, quien señala que el gobierno chino ha apoyado anualmente tanto al Colegio Nueva Granada (30,000 dólares estadounidenses), como a la Universidad de los Andes (100,000 dólares estadounidenses). De su parte, el colegio en China envía profesores a Colombia y el gobierno chino paga los salarios. En Colombia se subsidian equipos e instalación del Aula.

El objetivo central del Aula "Confucio» es la enseñanza del idioma chino, desarrollar el conocimiento de la cultura china, y en el futuro hacer convenios o puentes entre las diferentes culturas. Así mismo, el Aula sirve de intermediaria para otorgar becas a colegios y universidades cada año, hacer intercambios para aprender chino y desde aquel país venir a estudiar español. De manera específica, las actividades que se realizan en el Aula "Confucio», 
según su director, son: clases de chino-mandarín, la Semana Cultural China que tiene lugar una o dos veces en el año, se recomiendan los mejores estudiantes para becas en la República Popular China, se invita a los estudiantes de universidades y colegios a participar en China en conferencias sobre chino-mandarín (este programa se denomina Chinese Bridge), se hacen intercambios de estudiantes y profesores, se participa en la organización del día del Instituto «Confucio» en septiembre 27 y se realizan los exámenes oficiales de suficiencia en idioma chino.

Lo más importante que ha realizado el Aula «Confucio» en Colombia es «que los jóvenes tengan más oportunidades para conocer China, aprender chino-mandarín y estudiar en China mediante becas», agrega nuestro interlocutor, Kevin Zhang. Él señala que también en el colegio Nueva Granada hay cuatro profesores chinos quienes manejan grupos de tres a quince estudiantes y en total este año, a 112 estudiantes.

Anteriormente, el mandarín era la séptima opción de enseñanza de un idioma extranjero en el Colegio Nueva Granada, ahora es la tercera opción después del inglés y francés y se está trabajando para ser la segunda opción, en uno o dos años. Aquí se empieza a enseñar chino desde el nivel de tercero de Primaria [...] De otra parte también realizamos el examen de chino-mandarín en cuatro colegios de Bogotá.

Los maestros también se sienten «embajadores» de su cultura y tradiciones, tal como nos lo manifestaron en su momento. Cabe agregar que recientemente se acaban de crear otras dos aulas «Confucio» en colegios de Bogotá.

\section{ENTIDADES CULTURALES Y ACADÉMICAS CREADAS POR CHINOS Y COLOMBIANOS}

\section{1) Centro China}

Centro China, el Centro de Estudios de China en Colombia, fue creado en 2007 con el fin de ser un puente entre Colombia y China al generar conocimiento 
directo, hacer gestión, formación, consultorías e investigaciones interdisciplinarias en las áreas de relaciones internacionales entre China-Colombia y ChinaAmérica Latina, y en las áreas de Cultura y Medicina Tradicional China. Centro China presta sus servicios a las empresas tanto privadas como estatales, universidades, entidades culturales, y otras instituciones colombianas y chinas. Este centro, cuyo carácter es primordialmente académico, cuenta con la más larga experiencia en la práctica y enseñanza de la medicina tradicional china en Colombia para personal de la salud. Fue la primera entidad privada que desarrolló los cursos de idioma chino en el país y la que, a través de las relaciones públicas internacionales, ha construido más lazos con entidades académicas en China. Sus fundadores son colombianos pero entre el personal académico siempre ha contado con ciudadanos chinos.

A lo largo de su existencia ha organizado cursos y conferencias sobre diferentes facetas de China, viajes culturales y temáticos, ha contribuido a la realización de diplomados en varias universidades del país y es entidad asociada con la Universidad Nacional de Colombia en la realización de la Cátedra China. Esta iniciativa consiste en ciclos permanentes de conferencias abiertas al público de manera gratuita con el propósito de acercar a China a los colombianos desde una perspectiva interdisciplinaria. Se trata de conferencias dictadas por expertos, académicos y diplomáticos desde diferentes áreas. La Cátedra China cuenta también con el respaldo de la Embajada de la República Popular China en Colombia, y el Banco de la República. Centro China está asociada al Grupo de Estudios Chinos de la Universidad Nacional, y también colabora con la Maestría en Medicina Tradicional China de esa entidad. Centro China es miembro de la Red Iberoamericana de Sinología.

\section{2) La Alianza Colombo-China}

La Alianza Colombo-China fue fundada en Bogotá en 2007 (bajo el nombre de Fundación Cultural Colombo-China) con el objetivo primordial de enseñar el idioma chino y también promover la cultura china y el intercambio

$100 \frac{\text { PRIMER SEMESTRE } 2016}{\text { MIGRACIÓN Y DESARROLLO NÚM. } 26}$ 
intercultural entre colombianos y chinos. Hoy día ofrece además de los cursos de idioma chino, cursos de español para ciudadanos chinos, cursos de cítara china o GuZheng, cursos de artes marciales así como becas para estudiar en China. Los profesores son de nacionalidad china pero el personal administrativo es colombiano.

\section{Asociaciones de Amistad Colombo-China CREADAS POR COLOMBIANOS}

\section{1) Asociación de la Amistad Colombo-China}

El objetivo central de la asociación es el intercambio cultural del pueblo colombiano con el pueblo chino. A esta asociación, que se constituyó en un puente de amistad entre los dos pueblos, se le atribuye haber logrado que el gobierno colombiano diera reconocimiento diplomático a la República Popular China en lugar de Taiwán. Varios de sus miembros originales fueron posteriormente funcionarios vinculados a la Embajada de Colombia en Beijing. El doctor Jaime Moreno, presidente de la asociación, nos relata:

[...] fundamos la Asociación porque era inconcebible que Colombia no tuviera relaciones con un país de más de novecientos millones de habitantes en 1977 y sí tuviera unas relaciones precarias de tipo comercial con Taiwán, dándole un status de Estado sin tenerlo, entonces montamos la asociación fundamentalmente con el ánimo de hacer un intercambio de carácter cultural, intelectual y demás.

Para poder existir de manera independiente, el doctor Moreno señala que:

Hicimos muchas actividades de financiación. Organizamos un partido de fútbol entre la Selección Nacional de China y la Selección de Colombia. El día del partido, ocurrió un pequeño incidente: la banda del Batallón Guardia Presidencial que nos facilitó la Presidencia de la República para tocar los himnos de cada 
país, tocó el himno de Taiwán y no el himno de la República Popular China; por supuesto, los jugadores no querían salir a jugar, tuvimos que hacer mil malabares para que salieran y al fin y al cabo el partido se llevó a cabo.

Posteriormente realizaron nuevas actividades mediante las cuales fue posible conseguir recursos y obtener una sede. Al respecto el doctor Moreno señala:

Con esos dineros que conseguimos en las primeras actividades compramos una casa en el sector bogotano tradicional de La Candelaria; allá duramos más o menos tres años, una casa absolutamente preciosa, pero el sector no se prestaba para las actividades tanto sociales, como intelectuales, razón por la cual decidimos vender y comprar esta oficina.

Más adelante el doctor Moreno explica:

Gracias a nuestro diligente accionar, Colombia rompió esa relación precaria con Taiwán y se iniciaron relaciones diplomáticas y comerciales con la República Popular China. Hoy nosotros seguimos manteniendo no la relación entre los Estados, pero si la relación entre los pueblos. En los treinta y siete años que llevamos de vida hemos enviado en viajes organizados a cinco mil personas a China de todas las clases, profesionales, comerciales e industriales. Antiguamente, cuando nosotros fundamos la asociación, se nos perseguía un poco porque se sospechaba que estuviéramos metidos en la corriente maoísta o algo así. A partir de la formación de la República Popular China en 1949, Occidente rompió relaciones con China y solamente quedaron 18 países con relaciones diplomáticas. Es entonces cuando a Mao Zedong y Zhou Enlai se les ocurrió la brillante idea de formar asociaciones de amistad que fueron rompiendo poco a poco con el bloqueo. Hoy no hay un solo país que no quiera tener relaciones con China porque al fin y al cabo China es la fábrica del mundo.

Hoy día la asociación está concentrada en programar viajes turísticos a China, anualmente realiza una semana académica sobre China y publica la revista Amigos de China.

$102 \frac{\text { PRIMER SEMESTRE } 2016}{\text { MIGRACIÓN Y DESARROLLO NÚM. } 26}$ 


\section{2) Organización para el Fomento de las Relaciones Colombia-China}

En 2013, producto de la labor realizada durante más de diez años en pro de las relaciones entre ambos países, un grupo de gestores y académicos colombianos conformó esta organización, la cual cuenta con el respaldo de la Asociación de Amistad del Pueblo Chino con el Extranjero, entidad creada por la cancillería china para impulsar desde hace seis décadas la diplomacia popular, establecer relaciones de amistad e impulsar los vínculos con diferentes países.

El presidente de la Organización para el Fomento de las Relaciones Colombia-China (OFRCC), el doctor Jairo Buitrago Ruiz, sostiene que:

La Organización es un puente entre Colombia y China que busca facilitar y afianzar los vínculos, respaldar y acompañar las iniciativas tendientes a acercar a los dos países en los ámbitos político, científico, académico y cultural porque nuestro punto de partida es que hay que romper la barrera del desconocimiento mutuo. Por esto es importante conocer en profundidad a este país, situado además en el nuevo centro de gravedad que representa el eje Asia-Pacífico. Los ojos del mundo miran a China y Colombia también debe ser capaz de analizar en profundidad los constantes cambios que atraviesa esta nación, detectar las oportunidades a tiempo, y estar más presentes y visibles en China; así como China debe estarlo en Colombia.

La Organización fomenta la promoción de los intercambios culturales, científicos, tecnológicos y educativos entre ambos países. Para ello, prepara y envía misiones culturales y temáticas para grupos con intereses específicos, por ejemplo, la formación de grupos de médicos y profesionales del área de la salud en Medicina Tradicional China; y apoya la realización de diversos cursos de conocimiento y profundización sobre China. La OFRCC, en su interés de difundir el conocimiento en profundidad sobre China, apoya la organización de la Cátedra China en Bogotá, facilita el acceso a instituciones educativas, artísticas y culturales de China, realiza asesoría y sensibilización cultural previos a viajes de turismo y excursiones, y apoya la publicación de 
documentos, boletines e investigaciones tendientes a generar lazos de conocimiento bilateral.

\section{Cámaras de Comercio}

Los lazos de «asociatividad» surgidos de la confianza, el origen, las prácticas y habilidades compartidas entre las distintas organizaciones, se expresan claramente en las palabras de otros de nuestros interlocutores:

[...] Afortunadamente acá hay dos cámaras de comercio con China. Son mis amigos, siempre nos apoyan para abrir las puertas en Colombia. La ventaja de la Cámara de Comercio e Integración es que ya tiene muchas oficinas en China, tiene ocho, y su relación con China es más profunda, no sólo es con provincia, sino con ciudades, con empresarios, para hacer negocio, me parece que es un excelente trabajo.

\section{1) Cámara de Comercio e Integración Colombo-China}

La Cámara de Comercio e Integración Colombo-China es una entidad de carácter binacional que promueve la integración y desarrolla actividades de fomento del comercio bilateral. La Cámara fomenta la cooperación entre los nacionales chinos y los colombianos, lo mismo que entre entidades y organismos de los dos países, con el fin de desarrollar proyectos conjuntos de trabajo mediante acuerdos y convenios de mutuo beneficio. Actualmente, cuenta con ocho sedes en diferentes provincias chinas, siendo una de las cámaras binacionales con mayor número de oficinas en China. Vale anotar las ciudades chinas en donde existen oficinas de la Cámara de Comercio e Integración: Shanghái, Shenzhen, Chengdu, Dalian, Tianjin, Xiamen, Qingdao y Xiangyang.

A comienzos de 2013, esta entidad creó, junto con un grupo de empresarios, la Cámara Asia-Latinoamérica de Industria y Comercio - Camerasia-

$104 \frac{\text { PRIMER SEMESTRE } 2016}{\text { MIGRACIÓN Y DESARROLLO NÚM. } 26}$ 
con el fin de aprovechar la plataforma existente y poder integrar los países latinoamericanos con países asiáticos, buscando construir tres pilares centrales: exportaciones, importaciones y empresas conjuntas:

Nosotros le vendimos a China 5,000 millones de dólares el año pasado y trajimos 10,000 millones de dólares; de esos 5,000 el 96\% corresponde a petróleo, carbón y ferroníquel. Nosotros hacemos muchos esfuerzos para promover la integración, por ejemplo, tenemos nuestra propia marca de café, que la tenemos posicionada en China, ingresamos las esmeraldas, ahora estamos trabajando con frutas y los licores también. Nosotros nos encargamos de la distribución de la revista China Hoy en Colombia.

La Cámara ha establecido gran número de convenios con las CCPIT's chinas, (por sus siglas en inglés Chambers of Commerce for the Promotion of International Trade). Las CCPIT's son instituciones del Estado, dependientes del Ministerio de Comercio Chino (Mofcom) encargadas de promover todas las actividades que aporten al desarrollo del comercio exterior. La Cámara también trabaja en asociación con universidades colombianas para promover la formación y prácticas profesionales en China.

\section{2) Cámara Colombo-China de Inversión y Comercio}

La Cámara de Comercio e Inversión (CCCHIC) es una organización binacional sin ánimo de lucro creada en 2010, cuyo principal objetivo es el de promover las relaciones entre Colombia y China en los ámbitos político, económico, comercial y cultural. La CCCHIC representa los intereses de sus afiliados ante los gobiernos de China y Colombia, y ante organizaciones nacionales e internacionales. Esta organización surge en el marco de Expo-Shanghái, con el apoyo de las asociaciones y gremios colombianos de la producción, en donde no hubo al principio una voluntad del gobierno, sino de empresarios que lograron reunir los recursos para poder tener un pabellón dedicado a Colombia. 
Las entidades colombianas artífices de su nacimiento fueron la Fundación Colombiana del Pacífico, la Asociación Nacional de Empresarios (Andi), el embajador en misión especial para Expo Shanghái 2010, la Federación Nacional de Comerciantes (Fenalco) y la Sociedad de Agricultores de Colombia (SAC), entre otros. La CCCHIC está dedicada a labores de promoción del comercio, la inversión y la cultura entre Colombia y la República Popular China. También asesora a todas aquellas empresas y empresarios colombianos que deseen establecer relaciones comerciales con China a través de la localización y recomendación de los servicios que prestan algunos de sus afiliados. Las asesorías se complementan con algunos seminarios, los cuales se enfocan en varios temas como ofrecer conocimientos sobre la cultura de negocios china y la identificación de oportunidades en el mercado chino. Los eventos tienen un enfoque de doble vía, así, las temáticas expuestas son de interés tanto para empresarios colombianos que desean establecer una relación comercial con China como para empresarios chinos establecidos en Colombia.

La cCCHIC es también el punto de contacto para las delegaciones y empresas chinas que buscan representantes o clientes para invertir en Colombia. Asimismo, la Cámara organiza anualmente el Festival de Primavera en conmemoración a la celebración del Año Nuevo chino. Tal como nos comentara un funcionario de la embajada china, el respeto, la igualdad en el trato y la cooperación son elementos que priman en sus relaciones con las dos cámaras existentes en Colombia. En realidad, ambas cámaras están enfocadas en áreas distintas de trabajo y cubren sectores diferentes. La Cámara de Comercio e Inversión (CCCHIC) está más enfocada en realizar eventos divulgativos con el auspicio de las más grandes empresas. La Cámara de Integración trabaja más de la mano con comerciantes.

\section{Conclusiones}

Se puede colegir que la historia de la migración china a Colombia registra cuatro momentos clave: El primero remite al siglo antepasado luego de que

$106 \frac{\text { PRIMER SEMESTRE } 2016}{\text { MIGRACIÓN Y DESARROLLO NÚM. } 26}$ 
migrantes chinos llegaron a Panamá (antigua provincia colombiana) para los trabajos rudos de construcción del ferrocarril. El segundo refiere a la reubicación de un buen número de ellos en ciudades como Barranquilla y Buenaventura. El tercer momento es agenciado por el gobierno chino y su programa de apoyo en actividades de gastronomía, constituyendo posteriormente los tradicionales restaurantes y pequeños comercios. Sin embargo, a partir de los últimos diez años se registra un aumento de población originaria de China en la medida en que se establecen en el país funcionarios y altos ejecutivos de empresas chinas transnacionales, orientadas a las telecomunicaciones, la exploración y explotación petrolera y a la industria automotriz. Puede decirse que la actividad laboral que desarrollan los inmigrantes ha variado grandemente a lo largo de estos años, teniendo en cuenta que aquellos que llegaron en el pasado instalaron «almacenes» o «supermercados», restaurantes y lavaderos de ropa, rubros en los que por lo demás no hace falta conocer demasiado la lengua del lugar de residencia.

Sin duda, el proceso de implantación de poderosas empresas estatales que comienzan a convertirse en empresas mixtas al recibir inyección de capital privado y con proyección transnacional, acentúa la tendencia a que la migración sea de mayor calificación. En tal sentido, hay una clara diferencia entre los chinos inmigrados del siglo XIX y xX con respecto a los de inicios del siglo xxI, en relación con sus niveles socioeconómicos y educativos. Estas características diferenciadas se traducen en una divergencia significativa, particularmente en dos sentidos: en términos del nivel de aprendizaje y dominio del idioma español, y en el grado de integración a la sociedad de residencia, a pesar de tratarse de una cultura bastante arraigada a sus propios patrones y tradiciones.

La existencia de organizaciones chinas en Colombia es relativamente reducida porque la inmigración china es reciente y no ha sido muy numerosa si se la compara con otros países de América Latina. Los factores que contribuyen a explicar esta situación se refieren a las políticas inmigratorias restrictivas en Colombia, a una política exterior fragmentada y contradictoria y a la situación de violencia que ha caracterizado a la historia del país. Las organizaciones chinas que logramos identificar son de distinto origen. 
Unas son esencialmente fundadas por chinos, otras se originan por iniciativas del gobierno de la República Popular; otras son chino-colombianas y otras más han surgido por interés de colombianos. En las organizaciones chinas es preciso anotar que entre ellas suele existir un alto espíritu de cooperación.

Por otra parte, es de anotar un hecho reciente sin precedentes que va a contribuir a incrementar los flujos de ciudadanos chinos a Colombia: en febrero de 2015, la Ministra de Relaciones Exteriores, María Ángela Holguín, anunció que los ciudadanos de China que posean visado Schengen o de Estados Unidos podrán ingresar a Colombia sin necesidad de tramitar otro visado. La firma de esta resolución busca atraer a los chinos que quieran visitar Colombia, pero también incluye a los ciudadanos indios, tailandeses y vietnamitas. Esta medida incentivará el turismo comercial y el flujo de empresarios que están interesados en Colombia, lo cual puede impactar la inmigración china a este país. Lo anterior será apoyado por el interés chino en incrementar las relaciones políticas, económicas y culturales con Colombia con miras a elevarlas a una de tipo «estratégico integral», lo cual, dentro de la clasificación china de las relaciones con los países latinoamericanos en el marco de su política exterior, implicará un mayor apoyo a sus connacionales que decidan establecerse en este país.

\section{Bibliografía}

Aglietta, M. y Y. Landry (2007), «La Chine Vers la Superpuissance», Económica Groupama, París.

Asociación de la Amistad Colombo-China (2008), «La presencia china en Colombia», Revista Amigos de China, Bogotá, núm. 7.

Cancillería de Colombia (2013), Decreto 834: Ingreso, Permanencia y Salida de los Extranjeros del Territorio Nacional, 24 de abril de 2013.

(2015), "Ciudadanos chinos que tengan visado Schengen o de los Estados Unidos, no deberán tramitar visa para ingresar a Colombia», Boletín de prensa, 2 de marzo.

$108 \frac{\text { PRIMER SEMESTRE } 2016}{\text { MIGRACIÓN Y DESARROLLO NÚM. } 26}$ 
CHAN, S. (1990), «European and Asian Inmigration into the United States in Comparative Perspective: 1820's to 1920's», en V. Yans-McLaughlin (ed.), Inmigration Reconsidered: History, Society and Policies, Nueva York, Oxford University Press.

CheCA-ArTAsu (2007), "Hacia una geografía de las primeras migraciones chinas en el Caribe», Revista Bibliográfica de Geografía y Ciencias Sociales, núm. 12.

FLEISER, Friederike (2012), «La diáspora China: un acercamiento a la migración china en Colombia», Revista de Estudios Sociales, núm. 42, Universidad de Los Andes, Bogotá.

García Márouez, Gabriel (2007), El amor en los tiempos del cólera, citado por ChecaArtasu, op. cit.

Gómez, Alcides (2014), "China: El resurgimiento de una potencia económica», ponencia presentada en la Cátedra China, Universidad Nacional de Colombia, Bogotá, 19 de febrero.

Gómez, Diana A. (2007), "China: el país más emigrante del mundo», Observatorio Virtual Asia-Pacífico, Universidad Jorge Tadeo Lozano, Bogotá, en http:// dianagomezdiaz.blogspot.com/2012/06/china-el-pais-mas-emigrante-el -mundo.html

(2007), «China: La mayor migración del mundo», en P. García y Z. Robles Sánchez (eds.), Asia hoy: Nuevos desafíos para Colombia, Bogotá, Fundación Friedrick Ebert.

(2008), «Crece presencia china en Colombia», El Espectador, Bogotá, julio 29 de 2008, en http://www.elespectador.com/impreso/internacional/ articuloimpreso-crece-presencia-colombia

(2010), «China: Una migración de dimensiones colosales», en Migraciones internacionales. Crisis mundial, nuevas realidades, nuevas perspectivas, Bogotá, Universidad Nacional de Colombia.

(2013), «China y su relación con América Latina: Una aproximación desde el poder blando", en J. I. Cortés (ed.), América Latina y el Caribe-China. Relaciones políticas e internacionales, México, Universidad Nacional Autonoma de Mexico. Marriaga, Natalia (2007), «La Colonia Fantasma: Crónicas de inmigrantes chinos en Bogotá», tesis de grado en Comunicación Social, Universidad Javeriana, Bogotá.

(2012), "Crónicas de China en Colombia: Esperando al señor Chang», China Files, 21 de noviembre de 2012, en http://china-files.com/es/ 
link/23628/cronicas-de-chinos-en-colombia-\%E2\%80\%9Cesperando-alsenor-chang\%E2\%80\%9D

Patiño, Germán (2006), "Chinos y japoneses», Revista Semana, Bogotá, 8 de octubre de 2006.

(2014), «El Dragón que entró a Colombia», Revista Semana, 9 de septiembre de 2014, en http://www.semana.com/economia/articulo/el-dragon-queentro-colombia/403420-3

Revista Semana (2012), "El hueco hacia EE.UU. ahora pasa por Colombia», Revista Semana, 8 de agosto de 2012, en http://www.semana.com/nacion/articulo/ el-hueco-hacia-eeuu-ahora-pasa-colombia/262580-3

Shicheng, Xu (2014), «Mi segundo viaje a Colombia», China Hoy, vol. LIX, núm. 24, 10 de enero de 2014. 